\title{
The Use of Auger Spectroscopy for the Elemental Analysis of Presolar Silicate Grains.
}

\author{
Christine Floss ${ }^{1}$
}

${ }^{1}$ Laboratory for Space Sciences and Dept. of Physics, Washington University, St. Louis, MO 63130, USA.

Presolar silicate grains, formed in the outflows of evolved stars and in the ejecta of stellar explosions (supernovae), survived the formation of the solar system and are found in low abundances in primitive extraterrestrial materials. They are recognized by their isotopic signatures, which differ substantially from those observed in other solar system materials. These grains can be studied in the laboratory to gain a better understanding of stellar evolution and nucleosynthesis of the elements. They also provide information about conditions in the stellar sources in which they formed and the environments they have traversed subsequent to formation, including the interstellar medium (ISM), the early solar nebula and the parent bodies of the meteorites in which they are found. The isotopic composition of a presolar grain provides information about its stellar origin [1], but additional elemental and/or mineralogical information can constrain the conditions under which a grain condensed, and is necessary for comparison with astronomical data [2].

Auger spectroscopy is a well-established surface analytical technique in the materials sciences, which takes advantage of the emission of electrons with characteristic energies (Auger electrons) from a sample irradiated by an electron beam. Like X-ray energies measured in an electron microprobe or scanning electron microscope, the kinetic energies of Auger electrons provide information about sample composition. Because Auger electrons originate only from the top few nanometers directly under the electron beam, the spatial resolution for Auger spectroscopy is primarily dependent on the primary electron beam diameter and is on the order of tens of nanometers, ideally suited for the elemental characterization of presolar silicates, which typically have diameters of 200-300 nm.

The development of Auger spectroscopy for the analysis of presolar grains is discussed by [3]. Specific analytical protocols were established for the analysis of presolar silicates to reduce the possibility of electron beam damage, which can occasionally produce artifacts in the Auger spectra. These include use of a low beam current, beam rastering over the grain of interest and acquisition of multiple specral scans that are added together to obtain a single Auger spectrum. Auger spectra are typically subjected to a 7point Savitsky-Golay smoothing and differentiation routine prior to peak identification (Fig. 1). Sensitivity factors for the major rock-forming elements have been determined from olivine and pyroxene standards in order to quantify data from the spectral measurements. In addition, highresolution (10-20 $\mathrm{nm}$ ) elemental distribution maps can provide detailed qualitative information about the distribution of elements within and around the grains of interest (Fig. 2).

The elemental compositions of over 400 O-rich presolar grains have been measured by Auger spectroscopy. The vast majority of the grains analyzed are ferromagnesian silicates. While some grains have stoichiometries consistent with either olivine or pyroxene, a significant fraction have nonstoichiometric compositions. Irradiation, shock and sputtering processes in the interstellar medium can induce changes in the structures, chemical compositions and porosities of the grains [4] and may play a role in transforming stoichiometric grains into grains with non-stoichiometric compositions. 
Alternatively, non-stoichiometric compositions may be due to condensation under kinetic conditions. Presolar silicates also have higher than expected Fe concentrations. Secondary alteration has played a role in some meteorites [e.g., 5], but most of the meteorites with high presolar silicate abundances show little evidence of alteration, suggesting that much of the Fe is primary, the result of non-equilibrium condensation of the grains in the stellar envelopes where they formed. These inferences are consistent with modeling work on the formation of dust in O-rich stars, which suggests that most silicate dust forms during short high mass-loss episodes that occur after thermal pulses in thermally pulsing AGB stars [6]. The stellar environment during these episodes will be highly variable, with strong stellar winds and rapid temperature drops, leading to grain formation under kinetic conditions.

\section{References}

[1] Nittler L. R. et al., Astrophys. J. 682 (2008), p. 1450.

[2] Henning T., Ann. Rev. Astron. Astrophys. 48 (2010), p. 21.

[3] Stadermann F. J. et al., Meteorit. Planet. Sci. 44 (2009), p. 1033.

[4] Carrez P. et al., Meteorit. Planet. Sci. 37 (2002), p. 1599.

[5] Floss C. and Stadermann F. J., Meteorit. Planet. Sci. 47 (2012), p. 1869.

[6] Gail H.-P. et al., Astrophys. J. 698 (2009), p. 1136.

[7] Floss C. and Stadermann F. J., Geochim. Cosmochim. Acta 73 (2009), p. 2415.

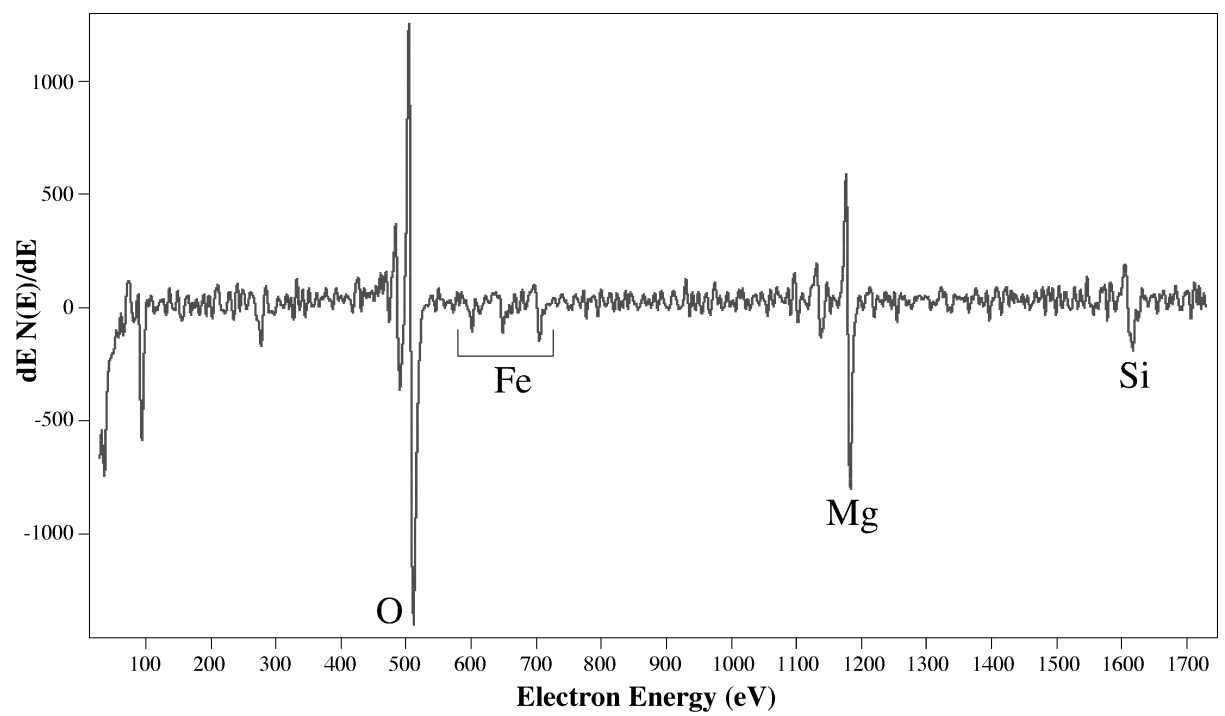

Figure 1. Differentiated Auger spectrum of a presolar ferromagnesian silicate from the CR3 chondrite QUE 99177 [7].
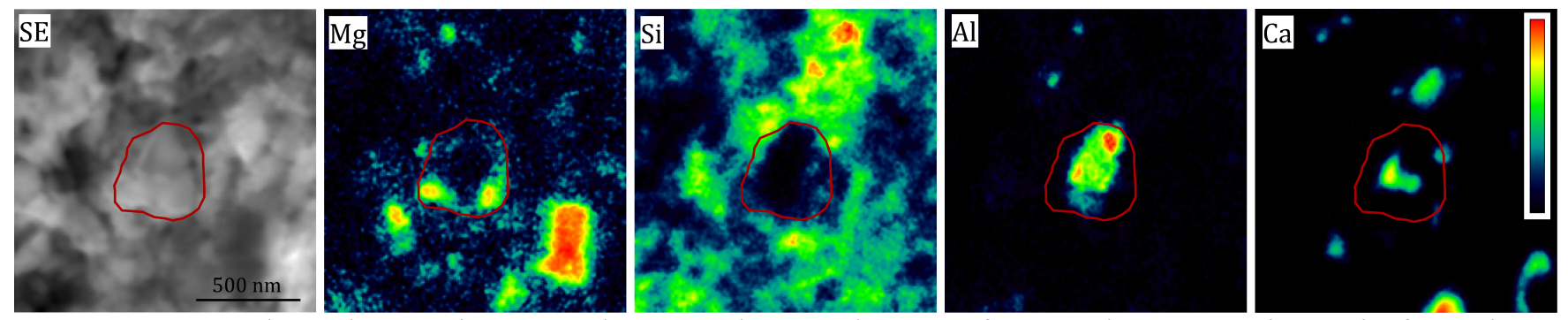

Figure 2. Secondary electron image and Auger elemental maps of a presolar composite grain from the Adelaide ungrouped carbonaceous chondrite [5]. 